\title{
Corrigendum: Inflammation and Tissue Remodeling in the Bladder and Urethra in Feline Interstitial Cystitis
}

\author{
F. Aura Kullmann ${ }^{1 *}$, Bronagh M. McDonnell ${ }^{1}$, Amanda S. Wolf-Johnston ${ }^{1}$, \\ Andrew M. Lynn ${ }^{1}$, Daniel Giglio ${ }^{2}$, Samuel E. Getchell ${ }^{1}$, Wily G. Ruiz ${ }^{1}$, Irina V. Zabbarova ${ }^{1}$, \\ Youko Ikeda ${ }^{1}$, Anthony J. Kanai ${ }^{1,3}$, James R. Roppolo ${ }^{3}$, Sheldon I. Bastacky ${ }^{4}$, \\ Gerard Apodaca ${ }^{1}$, C. A. Tony Buffington ${ }^{5,6}$ and Lori A. Birder ${ }^{1,3}$
}

\begin{abstract}
${ }^{1}$ Department of Medicine, School of Medicine, University of Pittsburgh, Pittsburgh, PA, United States, ${ }^{2}$ Department of Pharmacology, The Sahlgrenska Academy, Göteborg University, Göteborg, Sweden, ${ }^{3}$ Department of Pharmacology and Chemical Biology, School of Medicine, University of Pittsburgh, Pittsburgh, PA, United States, ${ }^{4}$ Department of Pathology, School of Medicine, University of Pittsburgh, Pittsburgh, PA, United States, ${ }^{5}$ Department of Medicine and Epidemiology, University of California, Davis, Davis, CA, United States, ${ }^{6}$ Department of Veterinary Clinical Sciences, The Ohio State University, Columbus, $\mathrm{OH}$, United States
\end{abstract}

Keywords: bladder, urethra, paraneurons, urothelium, von Brunn's nest

OPEN ACCESS

Approved by:

Frontiers In Systems Neuroscience

Editorial Office,

Frontiers Media SA, Switzerland

*Correspondence:

F. Aura Kullmann

aurakullmann@gmail.com

aurak@pitt.edu

Received: 06 October 2018 Accepted: 17 October 2018 Published: 08 November 2018

Citation:

Kullmann FA, McDonnell BM, Wolf-Johnston AS, Lynn AM, Giglio D, Getchell SE, Ruiz WG, Zabbarova IV, Ikeda Y, Kanai AJ, Roppolo JR, Bastacky SI, Apodaca G, Buffington CAT and Birder LA (2018)

Corrigendum: Inflammation and Tissue Remodeling in the Bladder and Urethra in Feline Interstitial Cystitis.

Front. Syst. Neurosci. 12:58, doi: 10.3389/fnsys.2018.00058

\section{A Corrigendum on}

Inflammation and Tissue Remodeling in the Bladder and Urethra in Feline Interstitial Cystitis by Kullmann, F. A., McDonnell, B. M., Wolf-Johnston, A. S., Lynn, A. M., Giglio, D., Getchell, S. E., et al. (2018) Front. Syst. Neurosci. 12:13. doi: 10.3389/fnsys.2018.00013

Daniel Giglio was not included as an author in the published article. The authors apologize for this error and state that this does not change the scientific conclusions of the article in any way. The original article has been updated.

\section{AUTHOR CONTRIBUTIONS}

FAK and LAB: designed the study and wrote the manuscript. FAK, BMM, AML, DG, SEG, ASWJ, WGR, IVZ, YI, JRR, SIB and GA: designed, collected and analyzed data. CATB: diagnosed and characterized the cats, and provided them for this study. AJK and LAB: supervised personnel.

Conflict of Interest Statement: The authors declare that the research was conducted in the absence of any commercial or financial relationships that could be construed as a potential conflict of interest.

Copyright (C) 2018 Kullmann, McDonnell, Wolf-Johnston, Lynn, Giglio, Getchell, Ruiz, Zabbarova, Ikeda, Kanai, Roppolo, Bastacky, Apodaca, Buffington and Birder. This is an open-access article distributed under the terms of the Creative Commons Attribution License (CC BY). The use, distribution or reproduction in other forums is permitted, provided the original author(s) and the copyright owner(s) are credited and that the original publication in this journal is cited, in accordance with accepted academic practice. No use, distribution or reproduction is permitted which does not comply with these terms. 